in vivo $35: 1057-1064(2021)$

doi:10.21873/invivo. 12350

\title{
Sorafenib and Lenvatinib Treatment for Metastasis/Recurrence of Radioactive Iodine-refractory Differentiated Thyroid Carcinoma
}

\author{
YASUHIRO ITO $^{1}$, NAOYOSHI ONODA ${ }^{1}$, TAKUMI KUDO ${ }^{2}$, HIROO MASUOKA ${ }^{1}$, \\ TAKUYA HIGASHIYAMA ${ }^{1}$, MINORU KIHARA ${ }^{1}$, AKIHIRO MIYA ${ }^{1}$ and AKIRA MIYAUCHI ${ }^{1}$ \\ Departments of ${ }^{1}$ Surgery, and ${ }^{2}$ Internal Medicine, Kuma Hospital, Kobe, Japan
}

\begin{abstract}
Background/Aim: Radioactive iodine-refractory differentiated thyroid carcinoma (RR-DTC) has been treated with multi-kinase inhibitors (MKIs), e.g., sorafenib (SOR) and lenvatinib (LEN). We analyzed the outcomes of RR-DTC patients who underwent SOR or LEN treatment at Kuma Hospital. Patients and Methods: We enrolled 21 and 18 patients treated with SOR and LEN, respectively. Results: The incidence of partial response in the LEN group was significantly higher than that in the SOR group. Serum thyroglobulin significantly decreased from the beginning of treatment to 1 month later in the LEN group (not in the SOR group). The neutrophil-lymphocyte ratio (NLR) was significantly decreased at 1 month later in both groups. An $N L R \geq 3$ at the start of MKI treatment had a prognostic impact. Conclusion: For RR-DTC, LEN could be more effective than SOR, at least in the short term. The first-line drug should be selected based on other factors (e.g., adverse events, patient background).
\end{abstract}

For distant metastasis of differentiated thyroid carcinoma (DTC) [papillary, follicular, and poorly differentiated thyroid carcinomas (PTC, FTC, and PDC)], radioactive iodine (RAI) therapy is the first-line treatment. However, the metastases are often RAI-refractory, and a portion of these metastases becomes progressive and life-threatening. At present in Japan, the multi-kinase inhibitors (MKIs), sorafenib (SOR) and lenvatinib (LEN), can be used to treat

This article is freely accessible online.

Correspondence to: Dr. Yasuhiro Ito, Department of Surgery, Kuma Hospital, 8-2-35 Shimoyamate-dori, Chuo-ku, Kobe, Hyogo 6500011, Japan. Tel: +81 783713721, Fax: +81 783713723, e-mail: ito01@kuma-h.or.jp

Key Words: Differentiated thyroid carcinoma, sorafenib, lenvatinib, prognosis. metastasis/recurrence of RAI-refractory differentiated thyroid carcinoma (RR-DTC). The targets of SOR and LEN differ significantly. For SOR, the targets are vascular endothelial growth factor receptor (VEGFR) 1-3, RET (including $\mathrm{RET} / \mathrm{PC}$ ), RAF (including BRAF) and platelet-derived growth factor receptor (PDGF) $\mathrm{b}(1,2)$. For LEN, the targets are VEGFR 1-3, RET, fibroblast growth factor receptor (FGFR) 1-4, KIT, and PDGFa (3-5). Both SOR and LEN have been shown to prolong patients' progression-free survival (PFS) in randomized phase III trials $(6,7)$, and treatment with LEN has also been shown to improve the overall survival (OS) of patients $>65$ years (8). Real-world data of RR-DTC treated with LEN or SOR have also been published (9-12).

It is important to appropriately select candidates for MKI therapy. In 2011, Miyauchi et al. showed that the thyroglobulin doubling-time (Tg-DT) had a significant prognostic impact for thyroglobulin-antibody (Tg-Ab)negative PTC patients who underwent a total thyroidectomy and thyrotropin suppression (13). They demonstrated that a low Tg-DT is a very strong prognostic factor for distant metastasis-free survival and cause-specific survival (CSS). In 2017, Sebra et al. demonstrated that the tumor volume doubling-time (TV-DT) was a significant prognostic marker predicting the CSS of patients (14). However, this DT has two major limitations: $(i)$ the DT becomes negative when the tumor volume or tumor marker decreases over time, which disturbs continuous analysis enrolling patients with positive and negative DT values; and (ii) the magnitude of the DT value is opposite to the magnitude of the tumor growth rate. These limitations can be solved by taking the inverse of DT (1/DT), and Miyauchi et al. proposed calling this index the doubling rate (DR) because it indicates the number of doublings that occur per unit time (15). At present, we regard patients with a Tg-DR and/or a TV-DR $>0.5$ as candidates and those with $>1$ as strong candidates for MKI treatment, although several other factors should be considered before the start of such treatment. 
Table I. Backgrounds and clinicopathological features of patients who were treated with SOR and LEN

\begin{tabular}{|c|c|c|}
\hline Variables & $\begin{array}{l}\text { SOR group } \\
\text { (21 patients) }\end{array}$ & $\begin{array}{l}\text { LEN group } \\
\text { (18 patients) }\end{array}$ \\
\hline \multicolumn{3}{|l|}{ Gender } \\
\hline Male & $7(33 \%)$ & $4(22 \%)$ \\
\hline Female & $14(67 \%)$ & $14(78 \%)$ \\
\hline Age (median) & $\begin{array}{c}54-87 \text { years } \\
(70 \text { years })\end{array}$ & $\begin{array}{c}29-81 \text { years } \\
\text { (73 years) }\end{array}$ \\
\hline \multicolumn{3}{|l|}{ Histology } \\
\hline Papillary & $18(86 \%)$ & $16(89 \%)$ \\
\hline Follicular & $3(14 \%)$ & 0 \\
\hline Poorly differentiated & 0 & $2(11 \%)$ \\
\hline \multicolumn{3}{|l|}{ Target organs } \\
\hline Lung or pleura & $14(67 \%)$ & $14(78 \%)$ \\
\hline Bone & $5(24 \%)$ & $2(11 \%)$ \\
\hline Others & $2(9 \%)$ & $2(11 \%)$ \\
\hline \multicolumn{3}{|l|}{ *Tg-DR before administration } \\
\hline$\leq 0.5$ (/year) & $3(14 \%)$ & $4(22 \%)$ \\
\hline$<0.5, \leq 1.0(/$ year $)$ & $4(19 \%)$ & $5(28 \%)$ \\
\hline$>1$ (/year) & $8(38 \%)$ & $5(28 \%)$ \\
\hline $\mathrm{Tg}-\mathrm{Ab}$ positive & $6(29 \%)$ & $4(22 \%)$ \\
\hline \multicolumn{3}{|c|}{ **TV-DR before administration } \\
\hline$\leq 0.5$ (/year) & $1(4 \%)$ & 0 \\
\hline$<0.5, \leq 1.0$ (/year) & $2(10 \%)$ & $4(11 \%)$ \\
\hline$>1$ (/year) & $9(43 \%)$ & $10(78 \%)$ \\
\hline Unevaluable or unknown & $9(43 \%)$ & $4(11 \%)$ \\
\hline \multicolumn{3}{|l|}{$* * *$ NLR at the beginning } \\
\hline$<3$ & $12(57 \%)$ & $11(61 \%)$ \\
\hline$\geq 3$ & $8(38 \%)$ & $7(39 \%)$ \\
\hline Unknown & $1(5 \%)$ & 0 \\
\hline Dosing period (median) & $\begin{array}{l}1-61 \text { months } \\
\text { (10 months) }\end{array}$ & $\begin{array}{l}1-44 \text { months } \\
\text { (16 months) }\end{array}$ \\
\hline Follow-up period (median) & $\begin{array}{l}1-74 \text { months } \\
\text { (38 months) }\end{array}$ & $\begin{array}{l}1-54 \text { months } \\
\text { (24 months) }\end{array}$ \\
\hline
\end{tabular}

*Tg-DR: Thyroglobulin doubling rate; **TV-DR: Tumor volume doubling rate; ***NLR: Neutrophil-to-lymphocyte ratio.

Based on the above-mentioned factors, we have strictly selected patients who are suitable for MKI therapy. We also considered other physical factors such as the patients' performance status (PS) and coexisting diseases, and social background factors including the patients' preference regarding treatment, their financial condition, occupation, and family support. In this study, we retrospectively investigated the outcomes of MKI therapy for RR-DTC at our institution in Kobe, Japan.

\section{Patients and Methods}

Our institution began providing MKI therapy for progressive distant metastasis/recurrence of RR-DTC in June 2014. At that time, only SOR was available for use. In December 2015, we started MKI therapy using LEN. Thereafter, LEN was more likely to be selected as the first-line treatment.

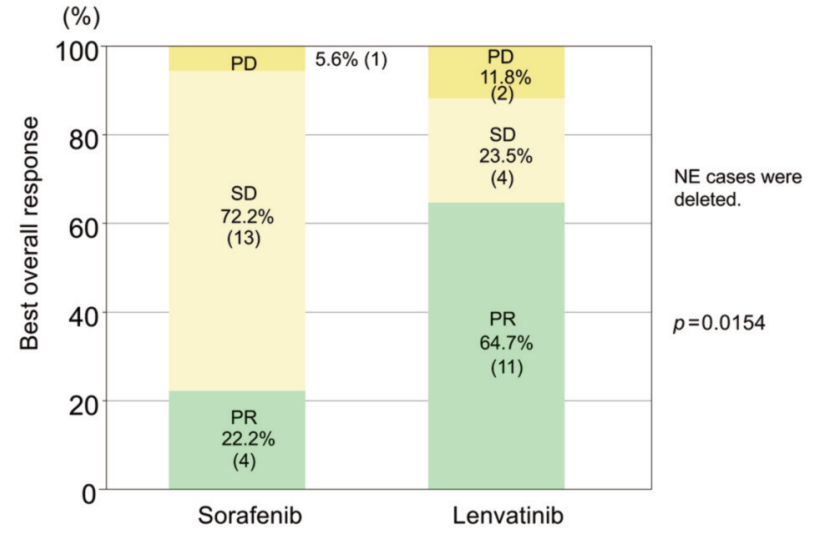

Figure 1. Best overall response of the sorafenib-and lenvatinib-treated patients with radioactive iodine-refractory differentiated thyroid carcinoma. PR: Partial response; SD: stable disease; PD: progressive disease; NE: not evaluable.

We enrolled 21 patients who were treated with SOR and 18 patients who were treated with LEN during the period from June 2014 and July 2020. All patients started their therapy with the full dose of SOR (800 $\mathrm{mg} /$ day) or LEN ( $24 \mathrm{mg} /$ day). The doses were reduced or the treatment was stopped as appropriate when an adverse event was observed. The MKI regimens were continued until the observation of disease progression or the appearance of uncontrollable adverse events, or in accord with the patient's request to stop treatment.

We performed a blood examination, including the measurement of $\mathrm{Tg}, \geq 1 \times /$ month. The neutrophil-to-lymphocyte ratio (NLR) was calculated as the ratio between neutrophils and lymphocytes. We evaluated the patient's Tg and NLR values at four time points: the beginning of treatment, 1 month later, at the minimal value, and at the time of disease progression. We evaluated the changes in metastatic/recurred lesions mainly by computer tomography (CT) $\geq 1 \times / 3$ months.

The Tg-DR and TV-DR were calculated using the doubling time, doubling rate $\&$ progression calculator as previously described $(9$, 11). For calculating the TV-DR, we selected one metastatic lesion that was observed to develop most rapidly on CT scans. In each examination, the lesion's maximum diameter (D1) and the diameter in the direction perpendicular to the maximum diameter (D2) were measured. The tumor volume was then calculated using the ellipsoid equation $(\pi / 6 \times \mathrm{D} 1 \times \mathrm{D} 2 \times \mathrm{D} 2)$. Time $(\mathrm{T})$ is the time interval between the tumor's presentation and its measurement.

We then calculated the Tg-DR and TV-DR, which are the inverses of the Tg-DT and TV-DT, respectively. We used the data only when we were able to determine the $\mathrm{Tg}$ value and tumor volume $\geq 3$ times in sequential examinations. We did not calculate the Tg-DR for the Tg antibody-positive cases or the TV-DR for the cases with unmeasurable metastases.

We evaluated the patients' response to treatment by imaging studies (mainly CT) based on the Response Evaluation Criteria in Solid Tumors (RECIST) criteria (ver 1.1): complete response (CR), partial response (PR), progressive disease (PD), stable disease (SD), and not evaluable (NE). Our hospital's institutional review board decided that this study did not require its approval, due to its design as a retrospective analysis. 
A

Tg level (ng/ml)

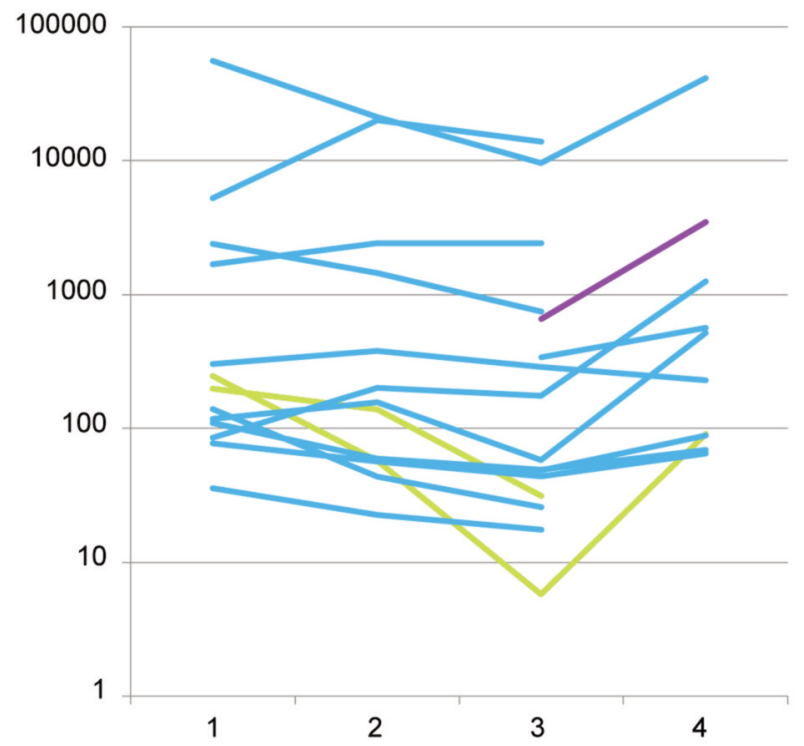

1. The beginning of administration

2. One month later

3. Minimal value

4. The time of disease progression
B

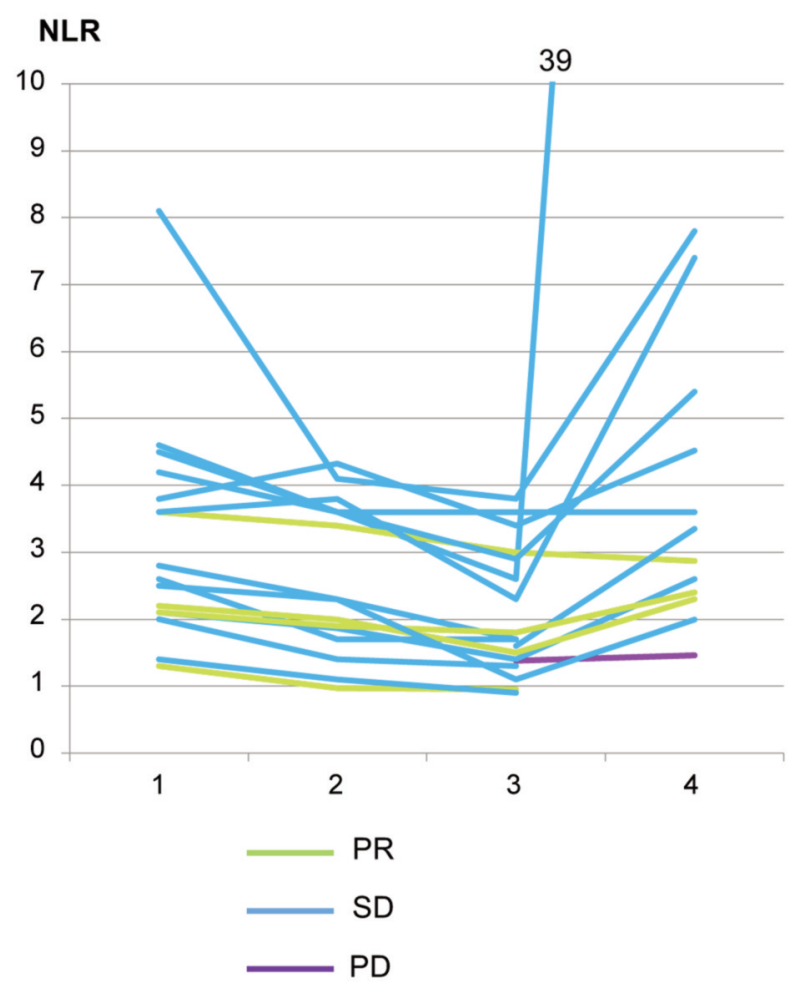

Figure 2. The changes in the Tg levels $(A)$ and $N L R(B)$ in the radioactive iodine-refractory differentiated thyroid carcinoma patients who were administered sorafenib. PR: Partial response; SD: stable disease; PD: progressive disease.

We used the software program StatFlex (Artec Co., Osaka, Japan) for the statistical analyses. Fisher's exact text and sign test were used to compare variables. For the time-sequence analysis, we used the Kaplan-Meier method with the log-rank test for univariate analysis. A $p$-value $<0.05$ was considered significant.

\section{Results}

We administered SOR and LEN to 21 and 18 patients (LEN and SOR groups), respectively. Table I summarizes the patients' backgrounds and clinicopathological features. The treatment of three patients was converted from LEN to SOR (two in our hospital and one in another hospital), and the treatment of five patients was converted from SOR to LEN at other hospitals. The values of both the Tg-DR and the TV-DR were high in large proportions of patients in both the SOR and LEN groups.

Figure 1 illustrates the best overall response of the patients in both groups. The best overall response could not be evaluated in one patient in the LEN group and in three patients in the SOR group. These patients were classified as NE and deleted from the analysis. The PR rate in the LEN group was significantly higher than that in the SOR group ( $p=0.0154)$. None of the patients were classified as having achieved a CR.

We next investigated the changes in the Tg levels and the NLR of the patients over time. We deleted the data of the four NE patients, and for the $\mathrm{Tg}$ analysis, the $\mathrm{Tg} \mathrm{Ab}$-positive patients were also deleted. Figure $2 \mathrm{~A}$ shows the changes in the Tg levels of the SOR-treated patients. The Tg data were collected for 14 patients both at the beginning of treatment and 1 month later. At 1 month after the start of treatment, compared to the value at the beginning of treatment, nine of these 14 patients $(64 \%)$ showed lower Tg levels, and the other five patients $(36 \%)$ showed even higher Tg levels. No significant difference was detected in $\mathrm{Tg}$ levels between these two time points $(r=5)$. The $\mathrm{Tg}$ value at the time of disease progression was available in 10 patients, and nine $(90 \%)$ of these patients showed a higher Tg level at the time of disease progression than the minimal value.

Figure 2B illustrates the changes in the NLR in the SORtreated patients. The NLR at both the beginning of treatment and 1 month later was available in 16 patients; 14 (88\%) of them had a lower NLR at 1 month of SOR treatment compared to the beginning of their treatment. The statistical 
A

Tg level (ng/ml)

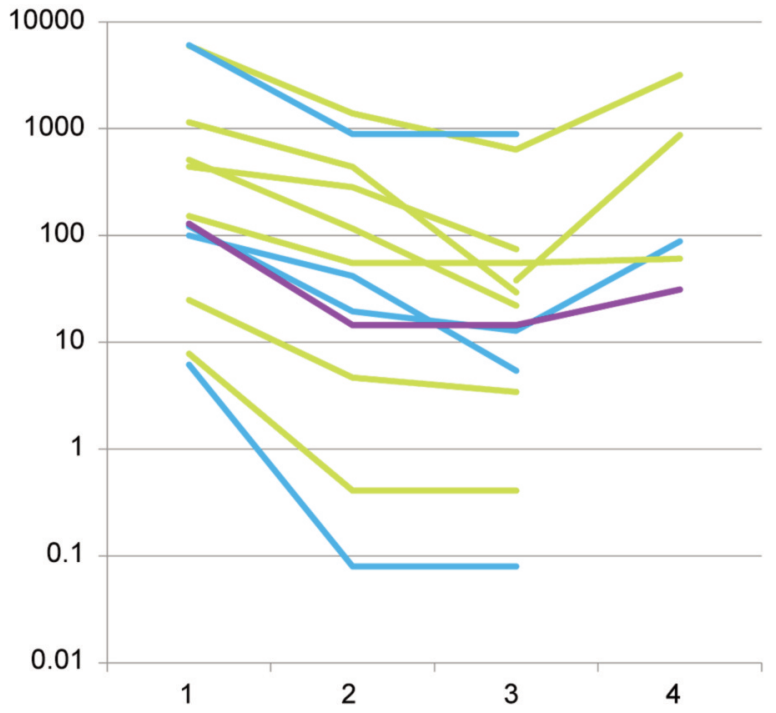

1. The beginning of administration

2. One month later

3. Minimal value

4. The time of disease progression
B

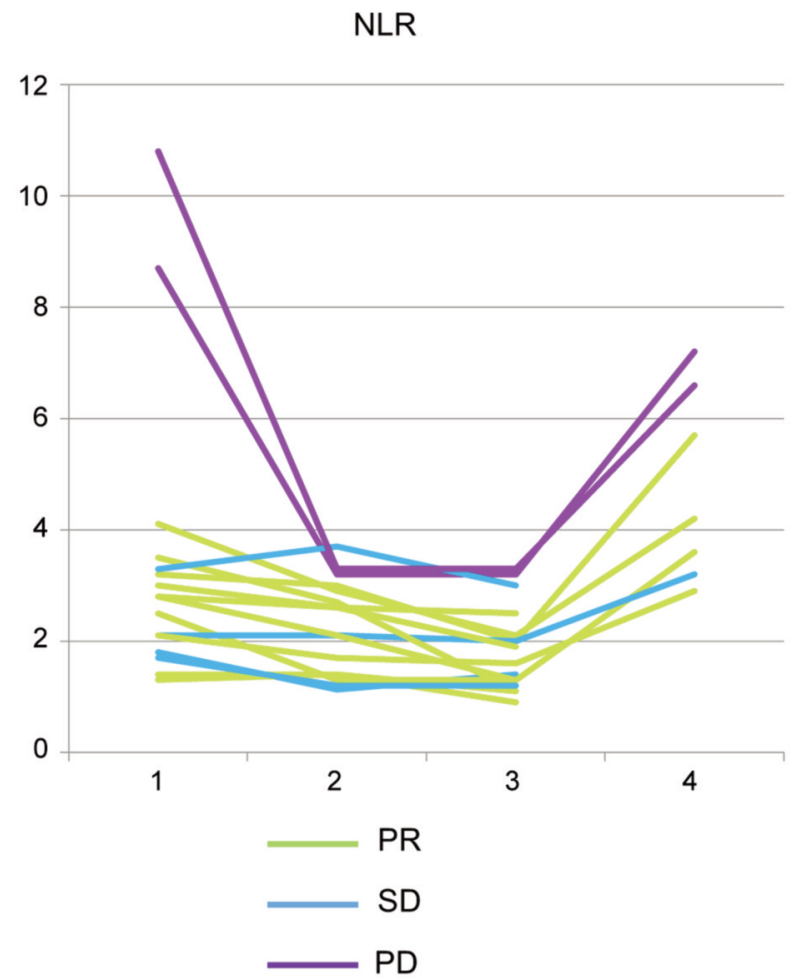

Figure 3. The changes in the Tg levels $(A)$ and neutrophil-lymphocyte ratio $(B)$ in the radioactive iodine-refractory differentiated thyroid carcinoma patients who were administered lenvatinib. PR: Partial response; SD: stable disease; PD: progressive disease.

Table II. Adverse events (all grades) of the SOR- and LEN-treated patients.

\begin{tabular}{llll}
\hline \multicolumn{1}{c}{$\begin{array}{c}\text { SOR } \\
(\mathrm{n}=21)\end{array}$} & & \multicolumn{2}{c}{$\begin{array}{c}\text { LEN } \\
(\mathrm{n}=18)\end{array}$} \\
\hline Hand-foot syndrome & $17(81 \%)$ & Hypertension & $17(94 \%)$ \\
Hypocalcemia & $8(38 \%)$ & Thrombocytopenia & $9(50 \%)$ \\
Proteinuria & $7(33 \%)$ & Hand-foot syndrome & $7(39 \%)$ \\
Diarrhea & $6(25 \%)$ & Proteinuria & $6(33 \%)$ \\
AST/ALT/ALP increased & $5(24 \%)$ & Creatinine increased, fatigue, hypocalcemia, appetite loss & $4(22 \%)$ \\
Hypertension & $4(20 \%)$ & Gingivitis, AST/ALT/ALP increased & $3(22 \%)$ \\
Eczema & $3(14 \%)$ & Dysgeusia & $2(11 \%)$ \\
Nausea/vomiting, gingivitis, creatinine increased, & $2(10 \%)$ & Bloody sputum, neck rigidity, arthralgia & $2(11 \%)$ \\
fatigue, hypophosphatemia, alopecia, CPK increased, & & & \\
inflammation & & & \\
Rhabdomyolysis, cystitis, dysgeusia & $1(5 \%)$ & & \\
\hline
\end{tabular}

analysis revealed that the NLR at 1 month later was significantly lower than that at the beginning $(\mathrm{r}=2, p<0.01)$. The NLR at the time of disease progression was collected in 13 patients, and 12 (92\%) of them showed an NLR higher than the minimal value.
Figure 3A, B shows the changes in the Tg and NLR values in the LEN-treated patients. The $\mathrm{Tg}$ at both the beginning of treatment and 1 month later was collected in 12 patients, and all of them showed a lower Tg level than that at the beginning of LEN treatment. The Tg values at 1 month 
A

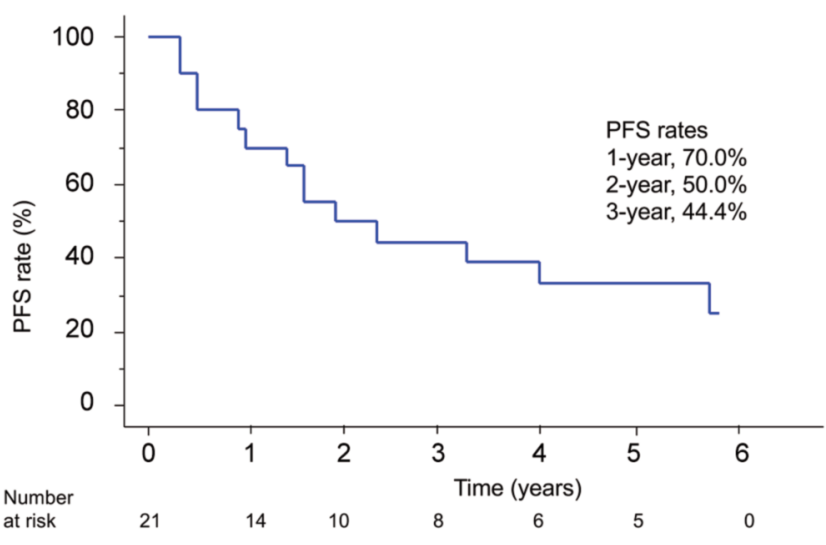

C

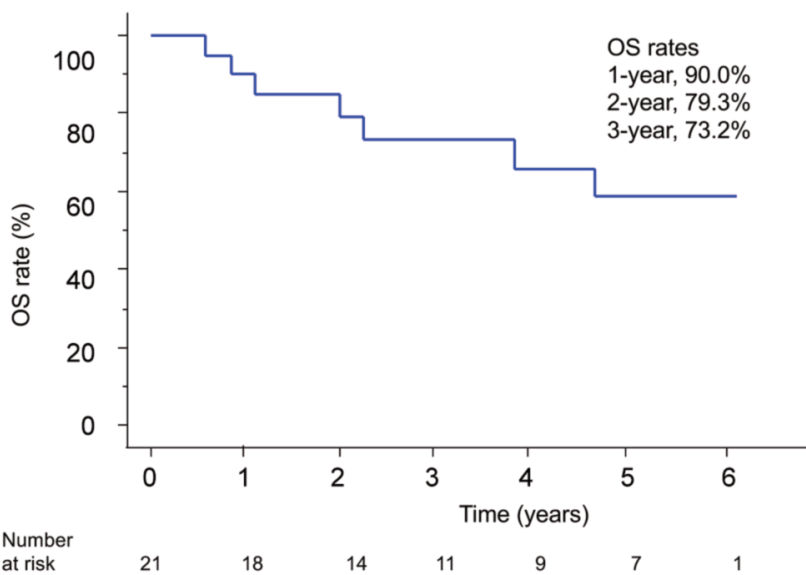

B

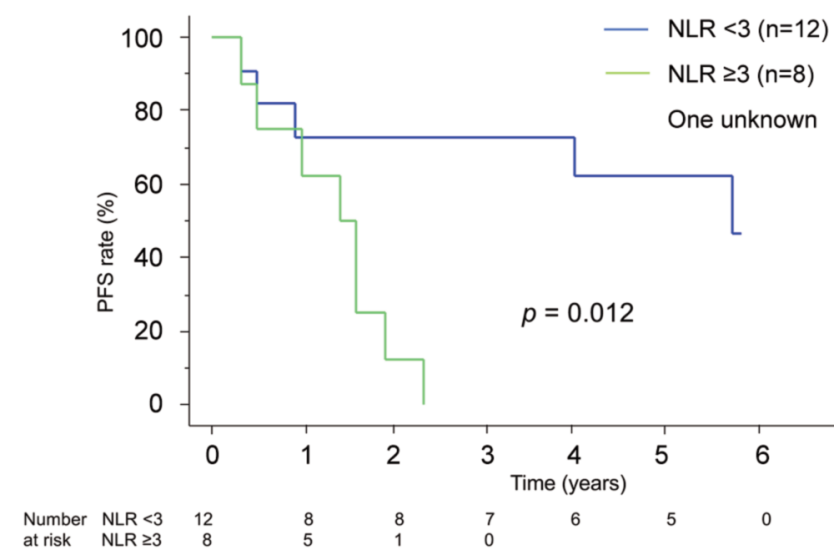

D

NLR $<3(n=12)$

NLR $\geq 3(n=8)$

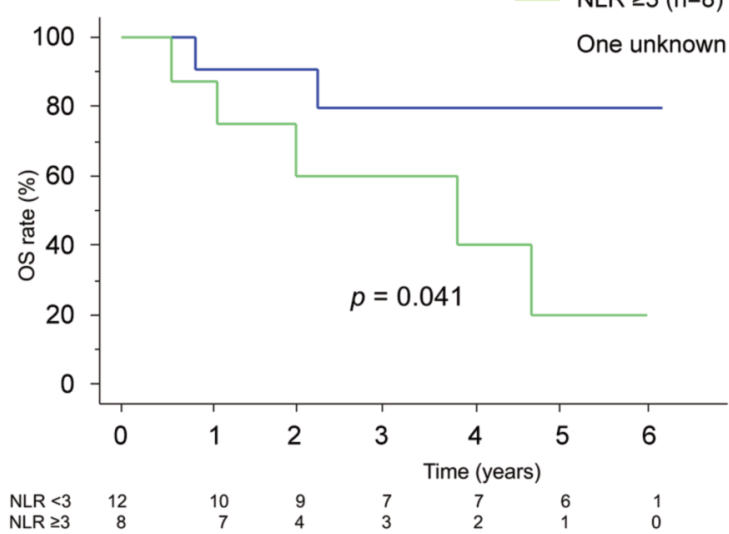

Figure 4. Prognosis of RR-DTC patients who were administrated sorafenib (SOR). A: Kaplan-Meier curve for the progression-free survival (PFS) of the RR-DTC patients who were administered SOR. B: Kaplan-Meier curves for the PFS of the SOR-treated patients with RR-DTC with a neutrophil-lymphocyte ratio $(N L R) \geq 3$ and those with an $N L R<3$. C: Kaplan-Meier curve for the overall survival (OS) of the radioactive iodinerefractory differentiated thyroid carcinoma patients who were treated with SOR. D: Kaplan-Meier curves for the OS of the SOR-treated patients with RR-DTC with an NLR $\geq 3$ and those with an NLR $<3$.

Table III. Adverse events ( $\geq$ grade 3 ) of the SOR-and LEN-treated patients.

$\begin{array}{cc}\substack{\text { SOR } \\(\mathrm{n}=21)} & \text { LEN } \\ (\mathrm{n}=18)\end{array}$

Hypocalcemia, hand-foot syndrome, hypertension AST/ALT/ALP increased, proteinuria, eczema Rhabdomyolysis, fatigue, appetite loss, CPK increased
$3(14 \%)$

$2(10 \%)$

$1(5 \%)$
Hypertension

Creatinine increased, proteinuria, hand-foot syndrome Gingivitis

AST/ALT/ALP increased, neck rigidity, eczema, appetite loss, thrombocytopenia
$15(83 \%)$

$3(17 \%)$

$2(11 \%)$

$1(5 \%)$ were significantly lower than those at the beginning $(\mathrm{r}=0$, $p<0.0001)$. The Tg level at disease progression was available for five patients, and all showed a higher Tg level at that time than the minimal Tg levels. The NLR at 1 month after the beginning of LEN treatment was lower than that at the beginning of treatment in $14(88 \%)$ of the 16 patients whose data could be collected. The NLR at 1 month later was significantly lower than that at the beginning of treatment 


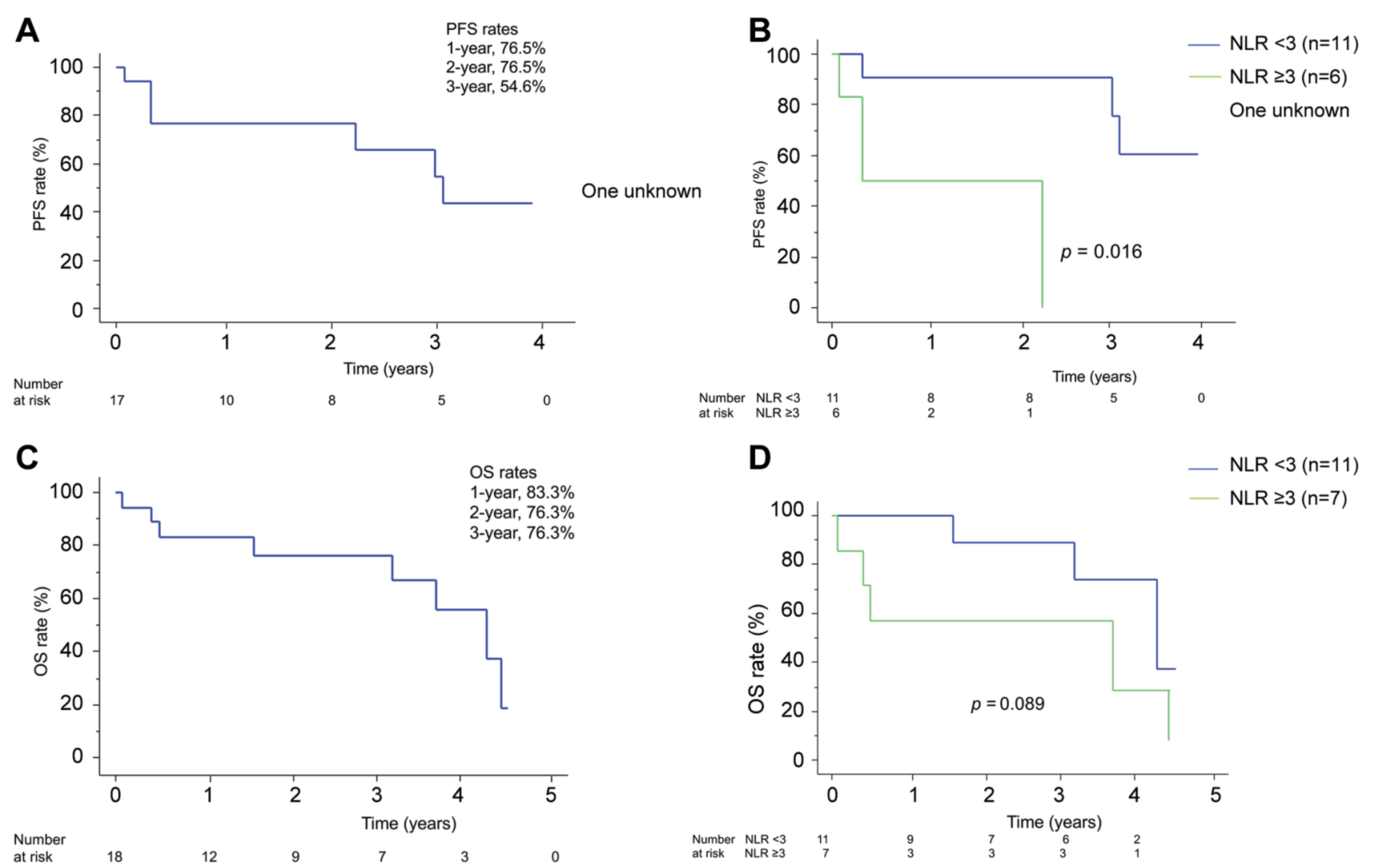

Figure 5. Prognosis of RR-DTC patients who were administrated lenvatinib (LEN). A: Kaplan-Meier curve for the progression-free survival (PFS) of the radioactive iodine-refractory differentiated thyroid carcinoma (RR-DTC) patients who were administered LEN. B: Kaplan-Meier curves for the PFS of the LEN-treated RR-DTC patients with an NLR $\geq 3$ and those with an NLR $<3$. C: Kaplan-Meier curve for the overall survival (OS) of the LEN-treated RR-DTC patients. D: Kaplan-Meier curves for the OS of the LEN-treated RR-DTC patients with an NLR $\geq 3$ and those with an NLR $<3$.

$(\mathrm{r}=2, p<0.05)$. The NLR at disease progression was collected for seven patients, and all of the NLRs were higher than the minimal value.

Adverse events that occurred in the SOR and LEN groups are shown in Tables II (all grades) and III (grade $\geq 3$ ). Handfoot syndrome (HFS) was detected in 17 patients $(81 \%)$ in the SOR group, but the incidence of HFS with grade $\geq 3$ was low at $14 \%$. The most common adverse event in the LENtreated patients was hypertension, which was detected in $94 \%$ of the patients. Eighty-three percent of the LEN-treated patients showed grade $\geq 3$ hypertension. Increased creatine, proteinuria, and HFS grade $\geq 3$ also occurred in $17 \%$ of the LEN-group patients.

The 1-, 2-, and 3-year progression-free survival (PFS) rates of the patients in the SOR group were $70.0 \%, 50.0 \%$, and $44.4 \%$, respectively, and the PFS of patients with an NLR $\geq 3$ at the beginning of treatment was significantly poorer than that of the patients with an NLR $\leq 3(p=0.012)$ (Figure 4A and B), The 1-, 2-, and 3-year overall survival (OS) rates of these patients was $90.0 \%, 79.3 \%$, and $73.2 \%$, respectively, and the patients with an NLR $\geq 3$ showed a significantly poorer overall survival (OS) rate than those with an NLR $<3 \quad(p=0.041)$ (Figure 4C and D).

In the LEN group, the 1-, 2-, and 3-year PFS rates were $76.5 \%, 76.5 \%$, and $54.6 \%$, respectively, and the patients with an NLR $\geq 3$ at the beginning of treatment showed significantly poorer PFS compared to the patients with an NLR $<3$ ( $p=0.016)$ (Figure 5A and B). The 1-, 2-, and 3-year OS rates of the LEN group patients were $83.3 \%, 76.3 \%$, and $76.3 \%$, respectively. The OS rate of the patients with an NLR $\geq 3$ at the start of treatment tended to be poor compared to that of those with an NLR $<3(p=0.089)$ (Figure 5C and D).

\section{Discussion}

In clinical settings in Japan, only two MKIs, SOR and LEN, can be administered for the treatment of RR-DTC. The results of our present analyses demonstrated the outcomes of RRDTC patients who were treated with SOR or LEN. As shown in Table I, the patients evaluated in this study tended to have 
aggressive disease based on high Tg-DR and TV-DR values; this is a result of the strict indications for MKI treatment that were adopted at our institution from the early period.

Considering the patients' best overall response, the PR rate achieved by the LEN-treated patients was higher than that of the SOR-treated patients, indicating that the tumors in the LEN group were more likely to shrink compared to those in the SOR group. We reported that a high Tg-DR (i.e., a short Tg-DT) significantly affected the prognosis of DTC patients (13). Although our knowledge about NLR remains fragmental, other research groups have reported the prognostic significance of a high NLR in DTC (16-18). In the present SOR group, although the NLR was significantly decreased at 1 month after the start of treatment, the Tg level did not change between the beginning of treatment and 1 month later. In the LEN group, in contrast, both the Tg and the NLR were significantly decreased at 1 month after the start of treatment compared to those at the beginning of treatment. In the present patient series, it was not prospectively decided whether SOR or LEN would be administered, but these findings suggest that LEN reduces the volume and growth activity of metastatic/recurred lesions more strongly than SOR, at least in the early phase.

Based on the above-described results, it could be appropriate to adopt LEN as the first-line treatment. However, both SOR and LEN have shown various types of adverse events, and these profiles differ significantly. For LEN, hypertension occurs at a very high rate, and $83 \%$ of the present LEN-treated patients suffered grade $\geq 3$ hypertension. In addition, grade $\geq 3$ proteinuria, increased serum creatinine, and HFS occurred in $17 \%$ of these patients. HFS is the most significant adverse event of SOR, but the incidence of grade $\geq 3$ HFS was $14 \%$ in the SOR group, which was even lower than that in the LEN group. Hypertension occurred also in the SOR group, but the incidence of grade $\geq 3$ hypertension was only $14 \%$. However, some of the SOR-treated patients suffered increases in AST/ALT/ALP (all grades: $24 \%$, and grade $\geq 3$ : $10 \%)$. It is therefore also important to select the first-line treatment by referring to the profiles of adverse events due to SOR and LEN.

In our series, the PFS rate in the LEN group was somewhat better than that in the SOR group, and the OS rate did not differ greatly between the two groups. However, since this was a retrospective analysis of a relatively small number of patients, we cannot directly compare the data of the present SOR and LEN groups. Fukuda et al. have shown that an NLR $\geq 3$ at the start of LEN treatment significantly affected the OS of DTC patients (16). Our present results also showed that an NLR $\geq 3$ at the beginning of treatment is a significant prognostic factor for the PFS and OS of SORtreated patients and for the OS of the LEN-treated patients. The PFS of the patients in the LEN group with an NLR $\geq 3$ tended to be poor, although the difference did not reach significance. We can thus conclude that a high NLR significantly reflects aggressive characteristics of RR-DTC, and it could be better to evaluate the NLR together with the Tg-DR and TV-DR when considering MKI treatment.

Our study has some limitations. It was a retrospective analysis and, in the early phase of the patient recruitment period, SOR was administered to all patients, whereas LEN is recently more likely to be chosen as the first-line treatment. The number of patients in our series is not large $(n=39)$. In addition, some patients were lost to follow-up.

In summary, for RR-DTC, lenvatinib is more effective (at least in the short term) than sorafenib, based on best overall response and the changes in the Tg level and NLR. However, the profiles of adverse events of sorafenib and lenvatinib treatment differ significantly, and this should be considered carefully when selecting the first-line drug.

\section{Conflicts of Interest}

Y. Ito and N. Onoda have received personal fees from Eisai and Bayer. The Authors declare no other conflicts of interest associated with this manuscript.

\section{Authors' Contributions}

Y. Ito wrote the manuscript. T. Kudo supported the data analyses. The other Authors contributed to the critical revision of the manuscript.

\section{References}

1 Wilhelm SM, Carter C, Tang L, Wilkie D, Mcnabola A, Rong H, Chen C, Zhang X, Vincent P, McHugh M, Cao Y, Shujath J, Gawlak S, Eveleigh D, Rowley B, Liu L, Adnane L, Lynch M, Auclair D, Tayler I, Gedrich R, Voznesensky A, Riedl B, Post LE, Bollag G and Trail PA: Bay 43-9006 exhibits broad spectrum oral antitumor activity and targets the RAF/MEK/ERK pathway and receptor tyrosine kinases involved in tumor progression and angiogenesis. Cancer Res 64(19): 7099-7109, 2004. PMID: 15466206. DOI: 10.1158/0008-5472.CAN-04-1443

2 Carlomagno F, Anaganti S, Guida T, Salvatore G, Giancarlo T, Wilhelm SM and Santoro M: BAY 43-9006 inhibition of oncogenic RET mutants. J Natl Cancer Inst 98(5): 326-334, 2006. PMID: 16507829. DOI: 10.1093/jnci/djj069

3 Matui J, Funahashi Y, Uenaka T, Watanabe T, Tsuruoka A and Asada M: Multi-kinase inhibitor E7080 suppresses lymph node and lung metastases of human mammary breast tumor MDAMB-231 via inhibition of vascular endothelial growth factorreceptor (VEGR-R) 2 and VEGF-R3 kinase. Clin Cancer Res 14(17): 5459-5465, 2008. PMID: 18765537. DOI: 10.1158/1078-0432.CCR-07-5270

4 Matsui J, Yamamoto Y, Funahashi Y, Tsuruoka A, Watanabe T, Wakabayashi T, Uenaka T and Asada M: E7080, a novel inhibitor that targets multiple kinases, has potent antitumor activities against stem cell factor producing human small cell lung cancer H146, based on angiogenesis inhibition. Int J Cancer 122(3): 664671, 2008. PMID: 17943726. DOI: 10.1002/ijc.23131 
5 Okamoto K, Kodama K, Takase K, Sugi NH, Yamamoto Y, Iwata $\mathrm{M}$ and Tsuruoka A: Antitumor activities of the targeted multi-tyrosine kinase inhibitor lenvatinib (E7080) against RET gene fusion-driven tumor models. Cancer Lett 340(1): 97-103, 2013. PMID: 23856031. DOI: 10.1016/j.canlet.2013.07.007

6 Brose MS, Nutting CM, Jarzab B, Elisei R, Siena S, Basthoit L, de la Fouchardiere C, Pacini F, Paschke R, Shong YK, Serman SI, Smit JWA, Chung J, Kappeler C, Rena C, Molnar I and Schlumberger MJ, DECISION investigators: Sorafenib in radioactive indine-refractory, locally advanced or metastatic differentiated thyroid cancer: A randomized, double-blind, phase 3 trial. Lancet 384(9940): 319-328, 2014. PMID: 24768112. DOI: $10.1016 /$ S0140-6736(14)60421-9

7 Schlumberger M, Tahara M, Wirth LJ, Robinson B, Brose MS, Elisei R, Habra MA, Newbold K, Shah MH, Hoff AO, Gianoukakis AG, Kiyota N, Taylor MH, Kim SB, Krzyzanowska MK, Dutcus CE, de las Heras B, Zhu J and Sherman SI: Lenvatinib versus placebo in radioiodine-refractory thyroid cancer. N Engl J Med 372(7): 621-630, 2015. PMID: 25671254. DOI: $10.1015 /$ NEJMoa1406470

8 Brose MS, Worden FP, Newbold KL, Guo M and Hurria A: Effect of age on the efficacy and safety of lenvatinib in radioiodine-refractory differentiated thyroid cancer in the phase III SELECT trial. J Clin Oncol 35(23): 2692-2699, 2017. PMID: 28613956. DOI: 10.1200/JCO.2016.71.6472

9 Masaki C, Sugino K, Saito N, Akaishi J, Hames KY, Tomoda C, Suzuki A, Matsuzu K, Uruno T, Ohkuwa K, Kitagawa W, Nagahama $\mathrm{M}$ and Ito K: Efficacy and limitations of lenvatinib therapy for radioiodine-refractory differentiated thyroid cancer: Real-world experiences. Thyroid 30(2): 214-221, 2020. PMID: 31854270. DOI: $10.1089 /$ thy.2019.0221

10 Wison L, Huang $\mathrm{W}$, Chen L, Ting $\mathrm{J}$ and Cao V: Cost effectiveness of lenvatinib, sorafenib and placebo in treatment of radioiodine-refractory differentiated thyroid cancer. Thyroid 27(8): 1043-1052, 2017. PMID: 28486081. DOI: 10.1089/thy. 2016.0572

11 Berdelou A, Borget I, Godbert Y, Nguyen T, Garcia M-E, Chougnet C, Ferru A, Buffet C, Chabre O, Huillard O, Leboullex S and Schlumberger M: Lenvatinib for the treatment of radioidinerefractory thyroid cancer in real-life practice. Thyroid 28(1): 72-78, 2018. PMID: 29048237. DOI: 10.1089/thy.2017.0205

12 Suzuki C, Kiyota N, Imamura Y, Goto H, Suto H, Chayahara N, Toyoda M, Ito Y, Miya A, Miyaushi A, Otsuki N, Nibu K and Minami H: Exploratory analysis of prognostic factors for lenvatinib in radioiodine-refractory differentiated thyroid cancer. Head Neck 41(9): 3023-3032, 2019. PMID: 3103380. DOI: 10/1002/head.25784
13 Miyauchi A, Kudo T, Miya A, Kobayashi K, Ito Y, Takamura Y, Higashiyama T, Fukushima M, Kihara M, Inoue H, Tomoda C, Yabuta $\mathrm{T}$ and Masuoka $\mathrm{H}$ : Prognostic impact of serum thyroglobulin doubling-time under thyrotropin suppression in patients with papillary thyroid carcinoma who underwent total thyroidectomy. Thyroid 21(7): 707-716, 2011. PMID: 21649472. DOI: $10.1089 /$ thy. 2010.0355

14 Sebra MM, Sherman EJ and Tuttle RM: Tumor volume doubling time or pulmonary metastases predicts overall survival and can guide the initiation of multikinase inhibitor therapy in patients with metastatic, follicular cell-derived thyroid carcinoma. Cancer 123(15): 2955-2964, 2017. PMID: 28369717. DOI: 10.1002/ cncr.30690

15 Miyauchi A, Kudo T, Ito Y, Oda H, Yamamoto M, Sasai H, Higashiyama T, Masuoka H, Fukushima M, Kihara M and Miya A: Natural history of papillary thyroid microcarcinoma: Kinetic analysis on tumor volume during active surveillance and before presentation. Surgery 165(1): 25-30, 2019. PMID: 30413323. DOI: $10.1016 /$ j.surg.2018.07.045

16 Fukuda N, Wang X, Ohmoto A, Yrasaki T, Sato Y, Nakano K, Nishizawa M, Yunokawa M, Ono M, Tomomatsu $J$ and Takahashi S: Sequential analysis of neutrophil-to-lymphocyte ratio for differentiated thyroid cancer patients treated with lenvatinib. In Vivo 34(2): 709-714, 2020. PMID: 32111774. DOI: 10.21873 /invivo.11828

17 Lee F, Yang P-S, Chien M-N, Lee J-J, Leung C-H and Cheng S$\mathrm{P}$ : An increased neutrophil-to-lymphocyte ratio predicts incomplete response to therapy in differentiated thyroid cancer. Int J Med Sci 15(14): 1757-1763, 2018. PMID: 30588200. DOI: 10.7150/ijms.28498

18 Gong W, Yang S, Yang X and Guo F: Blood preoperative neutrophil-to-lymphocyte ratio is correlated with TNM stage in patients with papillary thyroid cancer. Clinics (Sao Paulo) 71(6): 311-314, 2016. PMID: 27438563. DOI: 10.6061/clinics/ 2016(06)04 